\title{
Preliminary experimental study of urethral reconstruction with tissue engineering and RNA interference techniques
}

\author{
Chao Li, Yue-Min Xu and Hong-Bin Li
}

This study investigated the feasibility of replacing urinary epithelial cells with oral keratinocytes and transforming growth factor- $\beta 1$ (TGF- $\beta 1$ ) small interfering RNA (siRNA)-transfected fibroblasts seeded on bladder acellular matrix graft (BAMG) in order to reconstruct tissue-engineered urethra. Constructed siRNAs, which expressed plasmids targeting TGF- $\beta 1$, were transfected into rabbit fibroblasts. The effective siRNA was screened out by RT-PCR and was transfected into rabbit fibroblasts again. Synthesis of type I collagen in culture medium was measured by enzyme-linked immuno sorbent assay (ELISA). Autologous oral keratinocytes and TGF- $\beta 1$ siRNA-transfected fibroblasts were seeded onto BAMGs to obtain a tissue-engineered mucosa. The tissue-engineered mucosa was assessed morphologically and with the help of scanning electron microscopy. The TGF- $\beta 1$ siRNA decreased the expression of fibroblasts synthesis type I collagen. Oral keratinocytes and TGF- $\beta 1$ siRNA-transfected fibroblasts were seeded onto sterilized BAMG to obtain a tissue-engineered mucosa for urethral reconstruction. The compound graft was assessed using scanning electron microscope. Oral keratinocytes and TGF- $\beta 1$ siRNA-transfected fibroblasts had a good compatibility with BAMG. The downregulation of fibroblasts synthesis type I collagen expression by constructed siRNA interfering TGF- $\beta 1$ provided a potential basis for genetic therapy of urethral scar. Oral keratinocytes and TGF- $\beta 1$ siRNA-transfected fibroblasts had good compatibility with BAMG and the compound graft could be a new choice for urethral reconstruction.

Asian Journal of Andrology (2013) 15, 430-433; doi:10.1038/aja.2013.2; published online 1 April 2013

Keywords: RNA interference; tissue engineering; urethral reconstruction

\section{INTRODUCTION}

The treatment of complex long urethral stricture has always been one of the most difficult problems in urology surgery. In the previous studies, we built engineered oral mucosa tissue by inoculating oral mucosa cells on a cellular matrix of bladder submucosa. ${ }^{1,2}$ Although the effect was ideal within a short-term observation, we found that the collagen fibers were much more disordered as compared to that of the normal urethral submucosa tissue in the lower implanted oral mucosa tissue. It required further research on its long-term effect. There are two key problems in urethra repair: insufficient materials and urethral scar formation. However, these problems could be solved by improved engineering techniques to repair the urethra as well as effective control of collagen overproduction in the submucosa and inhibition of the formation of urethral scar.

The formation of urethra scar is an adverse clinical manifestation of excessive wound repair. It has been accepted that among the known cytokines, transforming growth factor- $\beta 1$ (TGF- $\beta 1$ ) is the most important stimulator for scar formation. ${ }^{3,4}$ To provide a theoretical basis for the therapy of complicated urethral strictures by genetic engineering and RNA interference (RNAi), we examined the effects of TGF- $\beta 1$ small interfering RNA (siRNA) on the expression of TGF$\beta 1$ and the secretion of type I collagen in rabbit fibroblasts. We successfully seeded oral keratinocytes and TGF- $\beta 1$ siRNA-transfected fibroblasts onto sterilized bladder acellular matrix graft (BAMG) in order to obtain a tissue-engineered mucosa for urethral repair. This would provide a better choice for tissue-engineered urethral reconstruction.

\section{MATERIALS AND METHODS}

Materials

The animal experimental protocol was approved by the Animal Care and Use Committee at our institution before initiation of the study. The modified Rheinwald and Green ${ }^{5}$ method for cutaneous keratinocytes was used for isolating and culturing oral keratinocytes. Materials were obtained from the following manufacturers: New Zealand white rabbits; Dulbecco's modified eagle's medium (DMEM; Gibco Invitrogen, Carlsbad, CA, USA); keratinocytes-SFM (KSFM; Gibco Invitrogen); fetal calf serum (Gibco Invitrogen); trypsin and EDTA (Gibco Invitrogen); Triton $\mathrm{X}-100$ and $\mathrm{NH}_{3} \cdot \mathrm{H}_{2} \mathrm{O}$ (Medicine Corporation of China, Shanghai, China); Dispase II enzyme (Roche, Munich, Germany); mitomycin (Gibco Invitrogen); 0.25\% chloromycetin; CFX Q-PCR instrument (Bio-Rad Laboratories, Shanghai, China); COL I-type enzyme-linked immunosorbent assay kit; and FAMsiRNA (GenePharma Co., Ltd, Shanghai, China).

\section{Methods}

BAMG preparation. The bladder taken from variant male rabbit of homogeneity was stripped of cells by being soaked in double distilled 
water for $24 \mathrm{~h}$ and further being soaked in a mixed solution (containing one liter of double distilled water, $2 \mathrm{ml} \mathrm{0.2 \%} \mathrm{Triton} \mathrm{X-100} \mathrm{and}$ $0.3 \mathrm{ml} \mathrm{NH}_{3} \cdot \mathrm{H}_{2} \mathrm{O}$ ) for 12 days, where the mixed solution was renewed every 3 days. After the procedure, BAMG was gained and reserved in $0.25 \%$ chloromycetin, followed by random hematoxylin and eosin (HE) and Masson staining to observe the effect of cell stripping. The BAMG was irradiated by ultraviolet ray for $12 \mathrm{~h}$ and poached by strokes of physiological saline solution before use.

Oral keratinocyte culture. The 10 -week-old male rabbit was intravenously anesthetized by $0.25 \%$ pentobarbitone and a small $(1.0 \times 0.4 \mathrm{~cm})$ buccal mucosa was taken. The buccal mucosa was digested by dispase II enzyme and the dermis was removed. Then oral keratinocytes were isolated from the epidermis after trypsinization. The oral keratinocytes were seeded into empty tissue culture dish and were cultured in KSFM. For expansion, oral keratinocytes were cultured and re-fed every 3 days until 85\%-95\% confluency (usually 1314 days).

Fibroblasts culture. Rabbit dermal tissue was excised and rinsed under sterile conditions. The tissue was placed in DMEM high glucose medium (plus the volume fraction of $10 \%$ fetal calf serum), cut into pieces and digested with Collagenase I. The cells were seeded into empty tissue culture dishes and were cultured in DMEM supplemented with $10 \%$ fetal calf serum. For expansion, the fibroblasts were cultured and re-fed every 3 days. The third-sixth generations of the cells were taken for experiments.

Transfection of siRNA. According to the TGF- $\beta 1$ sequence in the GeneBank, three pairs of FAM-TGF- $\beta 1-$ siRNA primers were designed as follows: (i) 5'-GGAACGGGCUCAACAUCUA-3' (the sense strand); $3^{\prime}$-CCUUGCCCGAGUUGUAGAU-5' (the anti-sense strand); (ii) 5' -CCUGCAAGUGCUCAAGUUA-3' (the sense strand); $3^{\prime}$-GGACGUUCACGAGUUCAAU-5' (the anti-sense strand); and (iii) $5^{\prime}$-GCGUCUAUAUGUUCUUCAA- ${ }^{\prime}$ ' (the sense strand) and
3'-CGCAGAUAUACAAGAAGUU-5' (the anti-sense strand). The construction of siRNA was completed by Biotechnology Co., Ltd. Three groups of siRNA (siTGF- $\beta 1-1$, siTGF- $\beta 1-2$, siTGF- $\beta 1-3$ ) were selected and transfected into rabbit fibroblasts. The effective siRNA was screened out by Q-PCR.

The most effective siRNA was transfected into the rabbit fibroblasts. The cells in a good growth state were subcultured after being trypsinized and were transfected with Lipofectamine 2000 (GenePharma Co., Ltd, Shanghai, China). The final siRNA concentration was set to be $100 \mathrm{nmol} \mathrm{l}^{-1}$. The fibroblasts were observed by fluorescence microscope after being transfected. The cell culture media were collected after being cultured for 1, 2, 3, 4, 5, 6 and 7 days. Synthesis of type I collagen was determined in the media by enzyme-linked immunosorbent assay (ELISA). Data were analyzed with Curve Expert 1.3 software.

Cell seeding. The passage 2 of the oral keratinocytes were isolated into cell suspension and were seeded onto BAMG with a density of $3 \times 10^{6} \mathrm{~cm}^{-2}$. The TGF- $\beta 1$ siRNA-transfected fibroblasts were seeded onto the other side of BAMG. The compound graft was cultured in an air fluid level for 1 week and the media was replaced every 3 days. The compound graft was assessed using HE staining and scanning electron microscopy.

\section{RESULTS}

After the cells were removed, BAMGs were essentially a porous membrane. HE and Masson staining demonstrated that there were no residual cells adhering to the grafts (Figure 1a and 1b). The oral keratinocytes showed a typical cobblestone shape (Figure 1c).

Gene expression changes were detected by relative quantification method, while the data were analyzed with $2^{-\Delta \Delta C T}$ method (Table 1). Compared to the control group, only the experimental group siTGF$\beta 1-1$ had a clear interference effect. The most effective siRNA (siTGF$\beta 1-1)$ was transfected into rabbit fibroblasts. ELISA results showed that the type I collagen concentration curve in the transfected cells was lower than that of the blank control groups. It meant that TGF- $\beta 1$
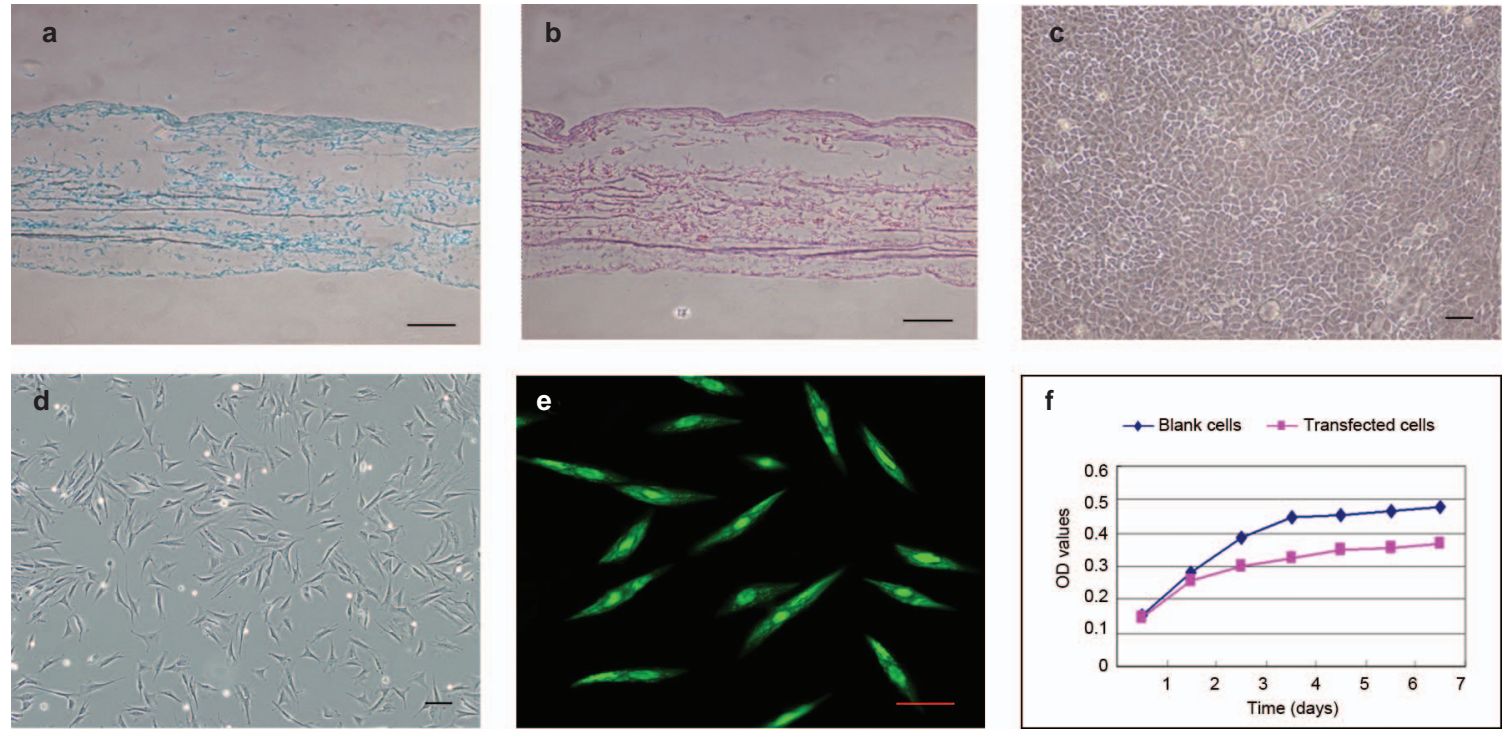

Figure 1 (a) BAMG Masson staining $(\times 40)$. (b) BAMG HE staining $(\times 40)$. (c) Passage 2 oral keratinocytes $(\times 100)$. (d) Passage 3 fibroblasts $(\times 100)$. (e) TGF- $\beta 1$ siRNA-transfected fibroblasts manifested green color fluorescence $(\times 200)$. (f) Type I collagen concentration curve in transfected cells was significantly lower than the blank control groups. Scale bar $=100 \mu \mathrm{m}$. BAMG, bladder acellular matrix graft; HE, hematoxylin and eosin; siRNA, small interfering RNA; TGF- $\beta 1$, transforming growth factor- $\beta 1$. 
Table 1 Gene expression changes detected by Q-PCR

\begin{tabular}{|c|c|c|c|c|c|c|c|}
\hline & $\begin{array}{l}\text { TGF- } \beta 1 C T \\
\text { values } 1\end{array}$ & $\begin{array}{l}\text { TGF- } \beta 1 C T \\
\text { values } 2\end{array}$ & $\begin{array}{l}\text { TGF- } \beta 1 C T \\
\text { values } 3\end{array}$ & $\begin{array}{l}\text { Internal reference } \\
\text { GAPDH CT values } 1\end{array}$ & $\begin{array}{l}\text { Internal reference } \\
\text { GAPDH CT values } 2\end{array}$ & $\begin{array}{l}\text { Internal reference } \\
\text { GAPDH CT values } 3\end{array}$ & $2^{-\triangle \Delta C T}$ values \\
\hline Blank control group & 20.22 & 19.90 & 19.88 & 13.72 & 13.76 & 13.79 & 1 \\
\hline siRNA control group & 20.78 & 20.22 & 20.92 & 14.92 & 14.67 & 15.01 & 1 \\
\hline siTGF- $\beta 1-1$ group & 22.63 & 23.66 & 22.92 & 14.79 & 14.39 & 14.57 & 0.179452 \\
\hline siTGF- $\beta 1-2$ group & 20.22 & 19.84 & 19.62 & 13.92 & 14.04 & 14.06 & 1.087994 \\
\hline siTGF- $\beta 1-3$ group & 20.37 & 20.22 & 20.14 & 14.05 & 13.96 & 14.49 & 0.953739 \\
\hline
\end{tabular}

Abbreviations: siRNA, small interfering RNA; TGF- $\beta 1$, transforming growth factor- $\beta 1$.

Gene expression changes were detected by relative quantification method, while data were analyzed with $2^{-\Delta \Delta C T}$ method. Compared to the control group, the experimental group siTGF- $\beta 1-1$ only has significant interference.

siRNA inhibited the expression of TGF- $\beta 1$ and reduced the secretion of type I collagen (Figure 1f). TGF- $\beta 1$ siRNA-transfected fibroblasts manifested a green color fluorescence (Figure 1d and 1e).

The passage 2 of oral keratinocytes were isolated into cell suspension and were seeded onto BAMG with a density of $3 \times 10^{6} \mathrm{~cm}^{-2}$. The TGF- $\beta 1$ siRNA-transfected fibroblasts were seeded onto the other side of BAMG with the same density. Scanning electron microscopy demonstrated that the oral keratinocytes had the shapes of ellipses and polygons and adhered to the grafts well. The cells formed pseudopodia that extended peripherally to allow the cells to connect with each other (Figure 2a). The TGF- $\beta 1$ siRNA-transfected fibroblasts also adhered to the grafts well (Figure $2 \mathbf{b}$ ).

\section{DISCUSSION}

Urethral strictures have been a reconstructive dilemma for many years due to the limited availability of tissue substitutes and the incidence of recurrence. Many tissues have been used for urethral reconstruction, including vascularized or free grafts of skin as well as buccal, lingual or colonic mucosa. ${ }^{6,7}$ However, the limited amount of autologous donor tissue limited these types of reconstruction, especially for the patients with long segment of strictures. Moreover, complications, such as recurrence, pain, swelling and oral opening limitation, often occur during the long-term follow-up. ${ }^{8}$ Tissue engineering is an effective method to solve these tough problems. ${ }^{9-11}$

In animal experiments, cell-seeded scaffolds have been successfully used to repair longer urethral defections in rabbit model. ${ }^{9}$ In our previous study, we built engineered oral mucosa tissue by inoculating the oral mucosa cells onto a cellular matrix of bladder submucosa. Although the effect was ideal within short-term observation, we found
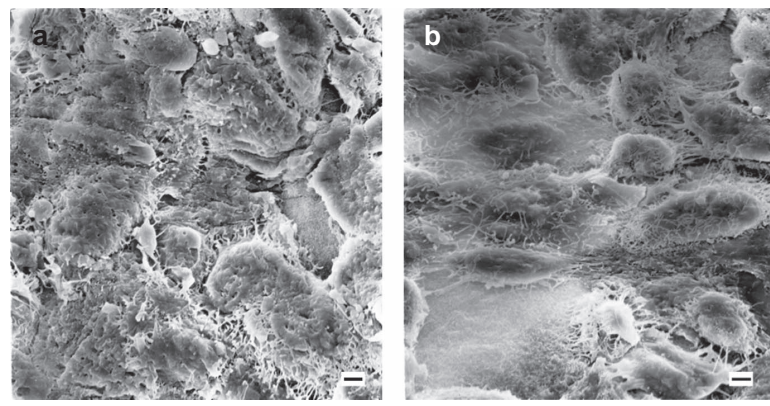

Figure 2 (a) Oral keratinocytes seeded onto the BAMG. The cells formed pseudopodia that extended peripherally to allow the cells to connect with each other. Scanning electron microscope $(\times 1000)$. (b) TGF- $\beta 1$ siRNA-transfected fibroblasts seeded onto the BAMG. The TGF- $\beta 1$ siRNA-transfected fibroblasts also adhered to the grafts well. Scanning electron microscope $(\times 1000)$. Scale bar $=10 \mu \mathrm{m}$. BAMG, bladder acellular matrix graft; siRNA, small interfering RNA; TGF- $\beta 1$, transforming growth factor- $\beta 1$. that the collagen fibers were much more disordered than normal urethral submucosa tissue in the lower implanted oral mucosa tissue.

Urethral scar is the main reason for urethral stricture recurrence. In 1993, Baskin et al. ${ }^{12}$ reported that the stenosis of urethra cavernosum was largely consisted of fibroblasts in the connective tissue. Morgia et $a l .{ }^{13}$ proved that the urethral stricture was at the lesion site of interstitial and was caused by the formation of large number of collagen tissue. There have been a number of studies on the inhibition of urethral scar overproduction. As a primary effector during wound healing, fibroblasts aid in healing by synthesis and secretion of collagen matrix. However, oversecretion of collagen will result in the deposition of a large number of type I collagen in the wound and ultimately the formation of hypertrophic scars. Therefore, an effective inhibition of collagen secretion could potentially inhibit excessive scar proliferation.

It is known that TGF- $\beta 1$ plays a key role ${ }^{14-19}$ in the process of wound repair including inflammation and extracellular matrix accumulation. ${ }^{20-22}$ The TGF- $\beta 1$ signaling is manifested by TGF- $\beta 1$ receptor activation, which leads to the phosphorylation of serine and threonine and activates the oversecretion of type I collagen. As a multifunctional growth factor, TGF- $\beta 1$ also stimulates the granulation tissue growth and repair. Therefore, TGF- $\beta 1$ has significant impact on wound healing and reconstruction of scars. ${ }^{23-25}$

RNAi is a form of posttranscriptional gene silencing that is mediated by short double-stranded RNA, known as siRNA. These siRNAs are capable of binding to a specific mRNA sequence and causing its degradation. The RNAi has been cultivated as a means of gene expression manipulation and is widely used in gene therapy research.

To some extent, RNAi substitutes for the technology of antisense oligonucleotides and gene knockout. This study was designed to detect the effect of TGF- $\beta 1$ on the secretion of type I collagen by transfecting TGF- $\beta 1$ siRNA into rabbit fibroblasts. The RNAi has its own unique specificity and timeliness. The selection of siRNA target sequence is one of the most important factors in the design of an effective siRNA. In this study, we synthesized three TGF- $\beta 1$ siRNAs and transfected them into the rabbit fibroblasts by a liposomal carrier. The siRNA with the best interference effect was screened out by the method of Q-PCR. We also detected type I collagen synthesis in the rabbit fibroblasts by enzyme-linked immunosorbent assay after being transfected with the TGF- $\beta 1$ siRNA. The results showed that the TGF- $\beta 1$ siRNA inhibited the expression of TGF- $\beta 1$ and might help to reduce the secretion of type I collagen and scar formation.

Taking oral mucosal cells along with the TGF- $\beta 1$ siRNA-transfected fibroblasts as seed cells and BAMG as the tent, we built the engineered oral mucosa in which excessive synthesis and secretion of type I collagen was inhibited by the tissue engineering and RNA interference. In this study, scanning electron microscopy demonstrated that the oral 
keratinocytes and the TGF- $\beta 1$ siRNA-transfected fibroblasts adhered well to the grafts and grew. Therefore, it not only promoted healing ability of the tissue-engineered urethra, but also inhibited the scar formation. This study provided a theoretical basis for reconstruction of the urethra and the decrease of urethral stricture recurrence rate.

\section{CONCLUSION}

In conclusion, we successfully seeded oral keratinocytes and TGF- $\beta 1$ siRNA-transfected fibroblasts onto the sterilized BAMG in order to obtain a tissue-engineered mucosa for urethral repair. This could provide a better choice for tissue-engineered urethral reconstruction. In our future studies, we are going to use the same methodology in an animal model of urethral stricture to test results in vivo.

\section{AUTHOR CONTRIBUTIONS}

CL participated in the design of the study, carried out the studies and drafted the manuscript. YMX participated in the design of the study. HBL carried out the studies. All authors read and approved the final manuscript.

\section{COMPETING FINANCIAL INTERESTS}

None of the authors had direct or indirect commercial financial incentive associated with publishing this article.

\section{ACKNOWLEDGMENTS}

This work was supported by the National Natural Science Foundation of China (No. 30901503).

1 Li C, Xu YM, Song LJ, Fu Q, Cui L et al. Urethral reconstruction using oral keratinocyte seeded bladder acellular matrix grafts. J Urol 2008; 180: 1538-42.

2 Li C, Xu YM, Song LJ, Fu Q, Cui L et al. Preliminary experimental study of tissueengineered urethral reconstruction using oral keratinocytes seeded on BAMG. Urol Int 2008; 81: 290-5.

3 Yu R, Cen Y. Transforming growth factor beta1/Smad3 signal transduction pathway and post-traumatic scar formation. Zhongguo Xiu Fu Chong Jian Wai Ke Za Zhi 2012; 26: 330-5.

4 Abe M, Yokoyama Y, Ishikawa O. A possible mechanism of basic fibroblast growth factor-promoted scarless wound healing: the induction of myofibroblast apoptosis. Eur J Dermatol 2012; 22: 46-53.
5 Rheinwald JG, Green $H$. Serial cultivation of strains of human epidermal keratinocytes: the formation of keratinising colonies from single cells. Cell 1975; 6 : 331-43

6 Song LJ, Xu YM, Hu XY, Zhang HZ. Urethral substitution using autologous lingual mucosal grafts: an experimental study. BJU Int 2008; 101: 739.

7 Patterson JM, Chapple CR. Surgical techniques in substitution urethroplasty using buccal mucosa for the treatment of anterior urethral strictures. Eur Urol 2008; 53 1162

8 Das SK, Kumar A, Sharma GK, Pandey AK, Bansal H et al. Lingual mucosal graft urethroplasty for anterior urethral strictures. Urology 2009; 73: 105.

9 Fu Q, Deng CL, Song XF, Xu YM. Long-term study of male rabbit urethral mucosa reconstruction using epidermal cell. Asian J Androl 2008; 10: 719-22.

10 Sade C, Ugurlu K, Ozcelik D, Huthut I, Ozer K et al. Reconstruction of the urethra defects with autologous fascial tube graft in a rabbit model. Asian J Androl 2007; 9 835-42.

11 Mangera A, Chapple CR. Tissue engineering in urethral reconstruction-an update Asian J Androl 2013; 15: 89-92.

12 Baskin LS, Constantinescu SC, Howard PS, McAninch JW, Ewalt DH et al. Biochemical characterization and quantitation of the collagenous components of urethral stricture tissue. J Urol 1993; 150: 642-7.

13 Morgia G, Saita A, Falsaperia M, Spampinato A, Motta M et al. Immunohistochemical and molecular analysis in recurrent urethral stricture. Urol Res 2000; 28: 319-22.

14 Rheinwald JG, Green H. Serial cultivation of strains of human epidermal keratinocytes: the formation of keratinising colonies from single cells. Cell 1975; 6 331.

15 Shek FW, Benyon RC. How can transforming growth factor beta be targeted usefully to combat liver fibrosis? Eur J Gastroenterol Hepatol 2004; 16: 123-6.

16 Qi Z, Atsuchi N, Ooshima A, Takeshita A, Ueno H. Blockade of type beta transforming growth factor signaling prevents liver fibrosis and dysfunction in the rat. Proc Natl Acad Sci USA 1999; 96: 2345-9.

17 de Gouville AC, Boullay V, Krysa G, Pilot J, Brusq JM et al. Inhibition of TGF-beta signaling by an ALK5 inhibitor protects rats from dimethylnitrosamine-induced liver fibrosis. Br J Pharmacol 2005; 145: 166-77.

18 George J, Roulot D, Koteliansky VE, Bissell DM. In vivo inhibition of rat stellate cell activation by soluble transforming growth factor beta type II receptor: a potential new therapy for hepatic fibrosis. Proc Natl Acad Sci USA 1999; 96: 12719-24.

19 Okuno M, Akita K, Moriwaki H, Kawada N, Ikeda K et al. Prevention of rat hepatic fibrosis by the protease inhibitor, camostat mesilate, via reduced generation of active TGF-beta. Gastroenterology 2001; 120: 1784-800.

20 Arias E, Anderson RN, Kung HC, Murphy SL, Kochanek KD. Deaths: final data for 2001. Natl Vital Stat Rep 2003; 52: 111-5.

$21 \mathrm{Kim} \mathrm{KH}$, Kim HC, Hwang MY, Oh HK, Lee TS et al. The antifibrotic effect of TGF-beta siRNAs in murine model of liver cirrhosis. Biochem Biophys Res Commun 2006; 343 1072-8.

22 Bottinger EP, Bitzer M. TGF- $\beta 1$ signaling in renal disease. J Am Soc Nephrol 2002 13: 2600-10.

23 Miyazono K. TGF-beta/SMAD signaling and its involvement in tumor progression. Bio Pharm Bull 2000; 23: 1125-30.

24 Dai $C$, Yang J, Liu Y. Transforming growth factor- $\beta 1$ potentiates renal tubular epithelia cell death by a mechanism independent of Smad signaling. J Biol Chem 2003; 278 . 12537-45.

25 Massague J. How cells read TGF-beta signals. Nat Rev Mol Cell Bio/2000; 1: 169-78. 